\title{
Patients Requiring Conversion to General Anesthesia during Endovascular Therapy Have Worse Outcomes: A Post Hoc Analysis of Data from the SAGA Collaboration
}

\author{
(D) C.Z. Simonsen, (D) S. Schönenberger, (DP.L. Hendén, (D)A.J. Yoo, (D). Uhlmann, (D)A. Rentzos, (D). Bösel, \\ (D). Valentin, and (D). Rasmussen
}

\begin{abstract}
BACKGROUND AND PURPOSE: Endovascular therapy for acute ischemic stroke is often performed with the patient under conscious sedation. Emergent conversion from conscious sedation to general anesthesia is sometimes necessary. The aim of this study was to assess the functional outcome in converted patients compared with patients who remained in conscious sedation and to identify predictors associated with the risk of conversion.
\end{abstract}

MATERIALS AND METHODS: Data from 368 patients, included in 3 trials randomizing between conscious sedation and general anesthesia before endovascular therapy (SIESTA, ANSTROKE, and GOLIATH) constituted the study cohort. Twenty-one (11\%) of 185 patients randomized to conscious sedation were emergently converted to general anesthesia.

RESULTS: Absence of hyperlipidemia seemed to be the strongest predictor of conversion to general anesthesia, albeit a weak predictor (area under curve $=0.62$ ). Sex, hypertension, diabetes, smoking status, atrial fibrillation, blood pressure, size of the infarct, and level and side of the occlusion were not significantly associated with conversion to general anesthesia. Neither age (mean age, $71.3 \pm 13.8$ years for conscious sedation versus $71.6 \pm 12.3$ years for converters, $P=.58$ ) nor severity of stroke (mean NIHSS score, $17 \pm 4$ versus $18 \pm 4$, respectively, $P=.27$ ) were significantly different between converters and those who tolerated conscious sedation. The converters had significantly worse outcome with a common odds ratio of $2.67(P=.015)$ for a shift toward a higher $\mathrm{mRS}$ score compared with the patients remaining in the conscious sedation group.

CONCLUSIONS: Patients undergoing conversion had significantly worse outcome compared with patients remaining in conscious sedation. No factor was identified that predicted conversion from conscious sedation to general anesthesia.

ABBREVIATIONS: $\mathrm{AUC}=$ area under the curve; $\mathrm{CS}=$ conscious sedation; $\mathrm{EVT}=$ endovascular therapy; $\mathrm{GA}=$ general anesthesia; $\mathrm{MABP}=$ mean arterial blood pressure; SAGA = SIESTA, ANSTROKE, and GOLIATH

$F$ ve studies published in 2015 proved the efficacy of endovascular therapy (EVT) for acute ischemic stroke caused by a large-vessel occlusion. ${ }^{1}$ However, numerous questions remain regarding how to best deliver this treatment, including evaluation of the optimal thrombectomy technique, ${ }^{2}$ the most effective method of patient

Received May 20, 2020; accepted after revision August 5.

From the Departments of Neurology (C.Z.S.) and Anesthesia (M.R.), Section of Neuroanesthesia, Aarhus University Hospital, Aarhus, Denmark; Department of Neurology (S.S.), Heidelberg University Hospital, Heidelberg, Germany; Departments of Anesthesiology and Intensive Care Medicine (P.L.H.), and Radiology (A.R.), Sahlgrenska Academy, University of Gothenburg, Sahlgrenska University Hospital, Gothenburg, Sweden; Division of Neurointervention (A.J.Y.) Texas Stroke Institute, Dallas-Fort Worth, Texas; Institute of Medical Biometry and Informatics (L.U.), University of Heidelberg, Heidelberg, Germany; Department of Neurology (J.B.), Klinikum Kassel, Kassel, Germany; and Department of Clinical Medicine, (J.V.), Danish Center for Clinical Health Services Research, Aalborg University and Aalborg University Hospital, North Denmark Region, Denmark. C.Z. Simonsen, S. Schönenberger, and P.L. Hendén contributed equally to this work.

Please address correspondence to Claus Z. Simonsen, MD, PhD' Department of Neurology, Aarhus University Hospital, Aarhus, Denmark; e-mail: clasim@rm.dk http://dx.doi.org/10.3174/ajnr.A6823 triage, ${ }^{3}$ or whether EVT should be performed with the patient under either general anesthesia (GA) or conscious sedation (CS).

Observational studies have suggested that EVT with the patient under CS is associated with better neurologic outcome and lower mortality compared with GA. ${ }^{4}$ However, 3 randomized trials reported similar outcomes between CS and GA..$^{5-7}$ Proposed benefits of CS include stable hemodynamics, clinical monitoring, and a potentially shorter procedure. The disadvantages are an unprotected airway and patient movement, which sometimes may require emergent conversion to GA. Patients who need conversion might be sicker (larger strokes, more medical complications), but the conversion procedure itself may also have a potentially deleterious influence on outcome due to the emergent anesthetic induction, associated hypotension, and added time delay before reperfusion.

Although most patients can be treated under the less complex CS, it is of interest to identify factors that can predict the risk of conversion and hence the requirement for GA. We undertook a detailed analysis of the patients who were converted from CS to 
Table 1: Reasons for conversion among the 21 patients who were randomized to conscious sedation but converted to general anesthesia

\begin{tabular}{lcccc}
\hline \multicolumn{1}{c}{ Reason for conversion (No.) } & SIESTA & ANSTROKE & GOLIATH & Total \\
\hline Severe agitation & 7 & 2 & 3 & 12 \\
Respiratory insufficiency/loss of airway & 3 & 1 & 1 & 5 \\
Direct puncture of the internal carotid artery & 0 & 4 & 0 & 4 \\
\hline
\end{tabular}

\section{Statistical Analysis}

We compared converters (CS to GA) with patients who remained in CS on all possible predictors. We used the Student $t$ test for variables of SDs or frequencies and percentages when appropriate. In addition, we conducted ordered logistic regression analysis on

GA in our individual patient data base from the 3 randomized trials to examine the outcome of the converted patients compared with patients who remained in CS. We also aimed to identify possible predictors associated with a need for GA with EVT.

\section{MATERIALS AND METHODS}

Access to original study data resulted from the cross-institutional SIESTA (Sedation versus Intubation for Endovascular Stroke TreAtment), ANSTROKE (Sedation Versus General Anesthesia for Endovascular Therapy in Acute Stroke-Impact on Neurological Outcome), and GOLIATH (General Or Local Anaestesia in Intra Arterial THerapy) Association (SAGA). ${ }^{5-7}$ The study database contains individual patient-level data concerning demographics, comorbidities, imaging and time metrics, anesthesia, and functional outcome for all patients included in the 3 trials. All recruited patients or their legal representatives had provided informed consent according to each trial protocol, and all trials had been approved by their respective local ethics committees. The decision for EVT was based on local treatment protocols. The 3 studies included patients with acute ischemic stroke caused by an occlusion in the anterior circulation that was eligible for EVT. The patients had to be 18 years of age or older and have an NIHSS score of $\geq 10$. Patients in the Anesthesia During Stroke (ANSTROKE) trial had to have a premorbid mRS of $0-3,{ }^{6}$ while in the General or Local Anesthesia in Intra Arterial Therapy (GOLIATH) trial, ${ }^{7}$ the premorbid mRS had to be $0-2$. GOLIATH also excluded patients with an infarct volume of $>70 \mathrm{~mL}$.

\section{Population}

The study population consists of all patients from the SAGA data originally randomized to CS.

\section{Outcome}

The outcome event is defined as conversion from CS to GA during EVT for any of the given reasons. The shift in the 90-day mRS score in the converted group was compared with the scores of the patients who remained in the CS group.

\section{Possible Predictors}

Common baseline and demographic factors for stroke were collected for all the patients: sex, age, hypertension, diabetes, hyperlipidemia, smoking status (Sedation vs Intubation for Endovascular Stroke Treatment [SIESTA] and GOLIATH: current smoker; ANSTROKE: current and previous smoker), atrial fibrillation, premorbid mRS score, NIHSS score on admission, ASPECTS, occlusion site, IV thrombolysis given, and mean arterial blood pressure (MABP) as well as systolic blood pressure at the start of EVT. the mRS at 90 days, comparing converters with nonconverters with a random effects on study identification. We estimated the group mean of mRS at 90-day follow-up for the 3 groups: the "converters," the patients who stayed in the CS group, and the GA group and compared relevant variables that might have affected outcome (time to groin puncture, minimum MABP, and proportion of successful reperfusion). Results were presented with $95 \%$ confidence intervals. In addition, we generated Grotta bars of the mRS score at 90-day follow-up stratified by the same groups.

Second, we conducted forward selection stepwise logistic regression without data splitting on the above-defined outcome with all possible predictors as input variables. Each variable was selected on the basis of the lowest $P$ value, and selection was based on a 3-fold cross validation. The optimistic incremental area under the curve (AUC) was estimated at each step, ie, without data split, as well as an incremental AUC based on the 3-fold cross-validation. In addition, we estimated the receiver operating characteristic curve for the final model, which was used to assess the predicted probabilities of conversion. Moreover, we estimated the AUC of a univariable logistic regression analysis of all selected potential predictors on the basis of a 3-fold cross-validation.

All analyses were conducted using STATA 15 (Release 15, 2017; StataCorp). A $P$ value $<.05$ was considered statistically significant.

\section{RESULTS}

The SIESTA study excluded 42 patients due to severe agitation and 7 due to vomiting/loss of airway before randomization. ${ }^{5}$ After randomization, 10 patients converted from CS to GA. (In the original article, 11 patients converted, but the patient who was converted due to puncturing of the carotid artery was only converted for the last few minutes of the procedure and was not counted as a converter in this analysis). ANSTROKE excluded 59 patients for medical reasons before randomization and 7 patients converted to GA after randomization. GOLIATH excluded 7 patients who were intubated before arrival at the EVT center, and 18 patients were excluded due to MR imaging contraindications, but only 3 of these were due to excessive movement or vomiting. Four patients were converted to GA after randomization. Conversion reasons are outlined in Table 1.

Of 185 patients randomized to CS, 21 (11\%) required conversion to GA. For the univariable analyses of associations, we found statistically significant differences for hyperlipidemia and premorbid mRS (Table 2). The absence of hyperlipidemia and a premorbid $\mathrm{mRS}$ of 0 were associated with a higher risk of conversion to GA.

Patients who converted from CS to GA scored significantly higher on the mRS at 90-day follow-up with an odds ratio of 2.67 $(P=.015)$ for a shift toward a higher score compared with the patients who remained in the CS group. Mean scores are presented 
Table 2: Among the 185 patients allocated to conscious sedation (CS), the 21 converters are compared to the 164 who were treated under CS

\begin{tabular}{lccc}
\hline & $\begin{array}{c}\text { CS as Treated } \\
(\boldsymbol{n}=164)\end{array}$ & $\begin{array}{c}\text { Converted to GA } \\
(\boldsymbol{n}=21)\end{array}$ & $\begin{array}{c}\boldsymbol{P} \\
\text { Value }\end{array}$ \\
\hline Hypertension & $96(59 \%)$ & $12(57 \%)$ & 1.00 \\
Diabetes & $28(17 \%)$ & $5(24 \%)$ & .54 \\
Hyperlipidemia & $72(44 \%)$ & $3(14 \%)$ & .009 \\
Smoker & $38(24 \%)$ & $3(14 \%)$ & .42 \\
Atrial fibrillation & $71(44 \%)$ & $10(48 \%)$ & .82 \\
Sex (male) & $85(52 \%)$ & $10(48 \%)$ & .82 \\
Pre-mRS ( $\geq 1)$ & $51(31 \%)$ & $2(10 \%)$ & .042 \\
ASPECTS (<6) & $22(14 \%)$ & $1(5 \%)$ & .48 \\
Left side affected & $79(48 \%)$ & $12(57 \%)$ & .49 \\
IV thrombolysis given & $49(30 \%)$ & $4(19 \%)$ & .44 \\
Occlusion type, ICA (neck) & $12(7 \%)$ & $0(0 \%)$ & .36 \\
ICA-T & $28(17 \%)$ & $4(19 \%)$ & \\
M1 & $83(51 \%)$ & $13(62 \%)$ & \\
M2 & $18(11 \%)$ & $0(0 \%)$ & \\
Tandem & $23(14 \%)$ & $4(19 \%)$ & .58 \\
Age (mean) (SD) (yr) & $71(14)$ & $72(12)$ & .27 \\
NIHSS score on admission (mean) (SD) & $17.3(3.8)$ & $17.8(4.4)$ & .55 \\
Systolic blood pressure start EVT (mmHg), & $165(28)$ & $161(25)$ & .62 \\
$\quad$ mean (SD) & & & $111(16)$ \\
MABP at start of EVT (mmHg), mean (SD) & $113(19)$ & & \\
\hline
\end{tabular}

Note:-ICA-T indicates ICA bifurcation; Pre, premorbid.

Table 3: Group mean of $\mathrm{mRS}$ at 90 -day follow-up, time to groin puncture, and minimum MABP for the 3 groups-the converters, the patients who stayed in the CS group, and the GA group

\begin{tabular}{|c|c|c|c|}
\hline & $\begin{array}{c}\text { Converted from CS } \\
\text { to GA (n=21) }\end{array}$ & $\begin{array}{l}\text { Stayed in CS } \\
(n=164)\end{array}$ & $\begin{array}{l}\text { GA Group } \\
(n=183)\end{array}$ \\
\hline Mean mRS (95\% Cl) & $4.3(3.7-4.9)$ & $3.1(2.8-3.4)$ & $2.8(2.5-3.1)$ \\
\hline $\begin{array}{l}\text { Mean rate of successful } \\
\text { reperfusion (mTICI } 2 \mathrm{~b}-3) \\
(95 \% \mathrm{Cl})\end{array}$ & $71.4(47.8-88.7)$ & $76.2(69.0-82.5)$ & $85.2(79.3-90.0)$ \\
\hline $\begin{array}{l}\text { Mean arrival at angiosuite to groin } \\
\text { puncture }(95 \% \mathrm{Cl})(\mathrm{min})\end{array}$ & $25.1(16.3-34.0)$ & 17.7 (15.7-19.8) & $24.0(21.9-26.2)$ \\
\hline $\begin{array}{l}\text { Mean groin puncture to } \\
\text { reperfusion }(95 \% \mathrm{Cl})(\mathrm{min})\end{array}$ & $90.2(68.8-111.6)$ & $67.2(59.1-75.3)$ & $63.1(55.7-70.4)$ \\
\hline $\begin{array}{l}\text { Mean onset to reperfusion } \\
(95 \% \mathrm{Cl})(\mathrm{min})\end{array}$ & $276(241-312)$ & $258(241-275)$ & $273(256-290)$ \\
\hline $\begin{array}{l}\text { Minimum MABP during EVT } \\
(95 \% \mathrm{Cl})(\mathrm{mm} \mathrm{Hg})\end{array}$ & $68.7(62.8-74.6)$ & $87.8(85.7-89.9)$ & $76.0(74.0-78.0)$ \\
\hline
\end{tabular}

Note:- $\mathrm{mTICl}$ indicates modified $\mathrm{TICl}$.

in Table 3, and outcomes are shown in Fig 1. The time of conversion was not registered, but many patients were probably converted after groin puncture, which is indicated by the longer procedural time in the converter group. However, there was no significant difference in the delay from onset to reperfusion (276 minutes [range, 241-312 minutes] for the converters versus 258 minutes [range, 241-275 minutes] for the CS group). The minimum MABP was significantly lower in the converter group compared with the CS group $(68.7 \mathrm{~mm} \mathrm{Hg}$ [range, $62.8-74.6 \mathrm{~mm} \mathrm{Hg}$ ] versus $87.8 \mathrm{~mm} \mathrm{Hg}$ [range, 85.7-89.9 $\mathrm{mm} \mathrm{Hg}$ ], respectively). The mean mRS score and rate of reperfusion were statistically favorable for the GA group compared with both of the remaining groups (Table 3 ).

Three variables were selected in the stepwise regression analysis, for which hyperlipidemia had the highest predictive value. Incremental AUCs of the selection process are shown in Table 4, and the receiver operating characteristic curve of the final model is shown in Fig 2.
The cross-validated AUC for hyperlipidemia was $0.62(95 \% \mathrm{CI}, 0.50-$ $0.73)$.

\section{DISCUSSION}

We performed an analysis of patients who converted from CS to GA in the SIESTA, ANSTROKE, and GOLIATH studies and had 2 main findings: First, patients who underwent emergent conversion from CS to GA had a significantly worse outcome than patients who had EVT under CS. Second, none of the tested variables were predictive of the risk of conversion (hence, the necessity for GA) except for the absence of hyperlipidemia. Indicators for the necessity of GA are generally considered to be stroke severity ${ }^{8}$ and laterality, with left-sided strokes more prone to need GA. ${ }^{9}$

Only the absence of hyperlipidemia was considered a predictor of conversion from CS to GA because the univariable AUC of the remaining potential predictors was $<0.6$. We believe that this is a chance finding. Also, the result should be interpreted with some caution because of the low event rate of the outcome. Other studies have not been able to confirm our findings, with 2 studies finding a near-significant higher frequency of hyperlipidemia in the CS arm, ${ }^{4,10}$ and another study finding a near-significant higher rate of hypercholesterolemia in the GA arm. ${ }^{11}$

The insignificance of our findings was further confirmed with a poor predictive power because the stepwise regression model conducted with data splitting was not able to display any predictive ability. Even the optimistic model, for which the AUC was calculated on the basis of the training set, barely showed a predictive ability above poor.

One large observational study looking at comorbidities among patients subjected to either CS or GA has not reported differences. ${ }^{12}$ A general finding in observational studies is that patients undergoing GA had higher NIHSS scores. ${ }^{13-15}$ The study by Campbell et $\mathrm{al}^{4}$ based on patients in the HERMES (Highly Effective Reperfusion evaluated in Multiple Endovascular Stroke Trials) collaboration found a median NIHSS score in the CS group of 17 versus 18 in the GA group, which was nearly significant, and a significantly lower ASPECTS (ie, bigger strokes) in the GA arm. This study found a higher prevalence of diabetes in the CS arm. (Another observational study found a higher frequency of diabetes in the GA arm. ${ }^{13}$ ) Age has, in some studies, been older in the GA group, ${ }^{11}$ but in others, it was older in the 


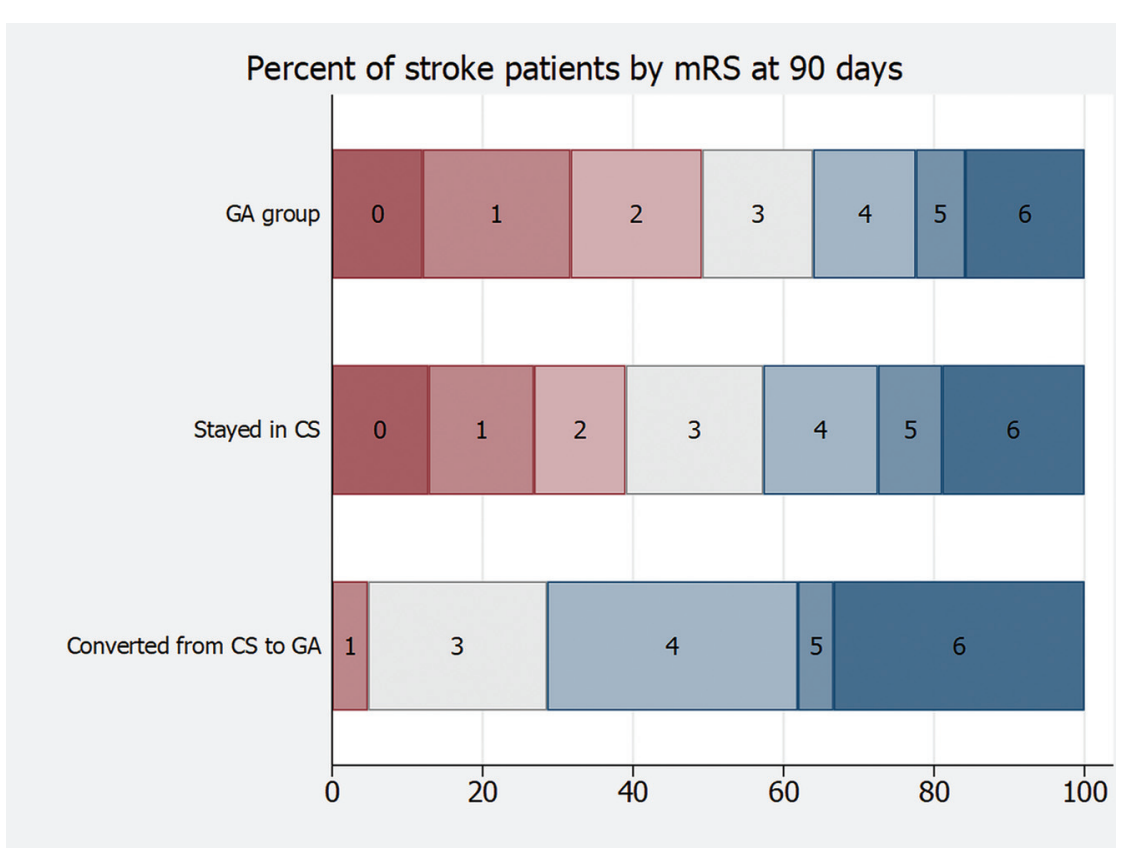

FIG 1. Grotta bars of the mRS score at 90 days by the 3 groups: the converters and the patients who stayed in the CS group and the GA group. The number in each bar indicates the mRS score. The odds ratio for a shift to a higher (worse) group was 2.67 for the converted group compared with the CS group.

Table 4: Incremental predictive value of the 4 best predictors of conversion from conscious sedation to general anesthesia ${ }^{a}$

\begin{tabular}{lccc}
\hline & $\begin{array}{c}\text { Incremental AUC } \\
\text { (without Data Split) }\end{array}$ & $\begin{array}{c}\text { Incremental AUC } \\
\text { (Cross-Validated) }\end{array}$ & $\begin{array}{c}\text { Univariable AUC } \\
\text { (Cross-Validated) }\end{array}$ \\
\hline $\begin{array}{l}\text { Hyperlipidemia } \\
\text { (95\% CI) }\end{array}$ & $0.65(0.56-0.73)$ & $0.62(0.50-0.73)$ & $0.62(0.50-0.73)$ \\
Diabetes $(95 \% \mathrm{Cl})$ & $0.68(0.58-0.78)$ & $0.66(0.54-0.78)$ & $0.49(0.36-0.61)$ \\
Pre-mRS $(95 \% \mathrm{CI})$ & $0.69(0.60-0.79)$ & $0.67(0.55-0.79)$ & $0.57(0.45-0.69)$ \\
Age $(95 \% \mathrm{Cl})$ & $0.73(0.62-0.83)$ & $0.62(0.50-0.74)$ & $0.38(0.26-0.50)$ \\
\hline
\end{tabular}

Note:-Pre indicates premorbid.

${ }^{a}$ Analysis was conducted without data splitting. Each AUC reflects the predictive power of the model consisting of the corresponding variable as well as all above variables.

${ }^{b}$ Not included in the final model.

CS group. ${ }^{4}$ Generally, the retrospective studies had large unbalanced patient groups, making identification of the role of differentiating characteristics very difficult.

We compared the converters with those in the CS group because this was the allocation from which the converters originated and hence the logical comparator. Because this was a randomized study, the GA group would, by chance, have a similar number of unstable patients, but they were not detected because they were intubated up front.

We found that the patients undergoing conversion had a significantly worse outcome compared with the patients remaining in the CS arm despite similar age and NIHSS and ASPECTS scores. We examined whether this could be explained by differences in time delay, reperfusion rates, or blood pressure drop associated with acute conversion. Reperfusion rates did not differ significantly between the converters and the CS group as shown in Table 3. Procedural time was longer for the converters, probably due to the conversion procedure, but there was no significant difference on the overall time from stroke onset to reperfusion, and this delay is unlikely to affect the outcome as shown in the meta-analysis. ${ }^{16}$

These findings argue for the conclusion that patients who need GA for medical reasons or because of excessive movement simply are at higher risk of a worse outcome. The combination of longer procedural time and lower MABP caused by the emergent conversion may have added to the poor outcome. The worse outcomes associated with GA in the observational studies are likely secondary to confounding by indication. This was also seen in the Interventional Management of Stroke (IMS-3) study. ${ }^{15}$ The strength of our analysis is that it is based on randomization.

In contrast to our findings, a recent study reported that conversion from local anesthesia/CS to GA was not associated with worse outcome. However, outcome in this study was very poor, with a median mRS score of 5 in both the converters and the GA and CS groups. ${ }^{17}$

In the SAGA individual patient data meta-analysis, the GA group proved to have a better outcome compared with the CS group. ${ }^{16}$ Due to the lack of predictor variables for conversion to GA in this study, it is impossible to perform a subgroup analysis to compare how the converters would have done with a primary GA versus primary CS strategy. Accordingly, it should be assumed that this subgroup should have better outcomes with primary GA, like the entire population. An alternative possibility is that this subgroup may help to explain why patients with primary GA did better overall. In either case, a reasonable implication is to perform primary GA on everyone to avoid hurting the approximately $10 \%$ of patients that would require conversion under a primary CS approach, provided that GA can be rapidly administered with attention to blood pressure control as in the SAGA trials.

The limitation of our study is that patients who were not able to undergo CS were excluded before randomization in the contributing trials. Another limitation is the relatively small number of patients in the group that required conversion to GA, reflecting the tolerance of most EVT patients for undergoing treatment under CS. However, the findings in the present study, particularly with respect to NIHSS and ASPECTS, may be because sicker patients who required GA were removed before randomization (ie, not eligible for both CS and GA) in the randomized trials. 


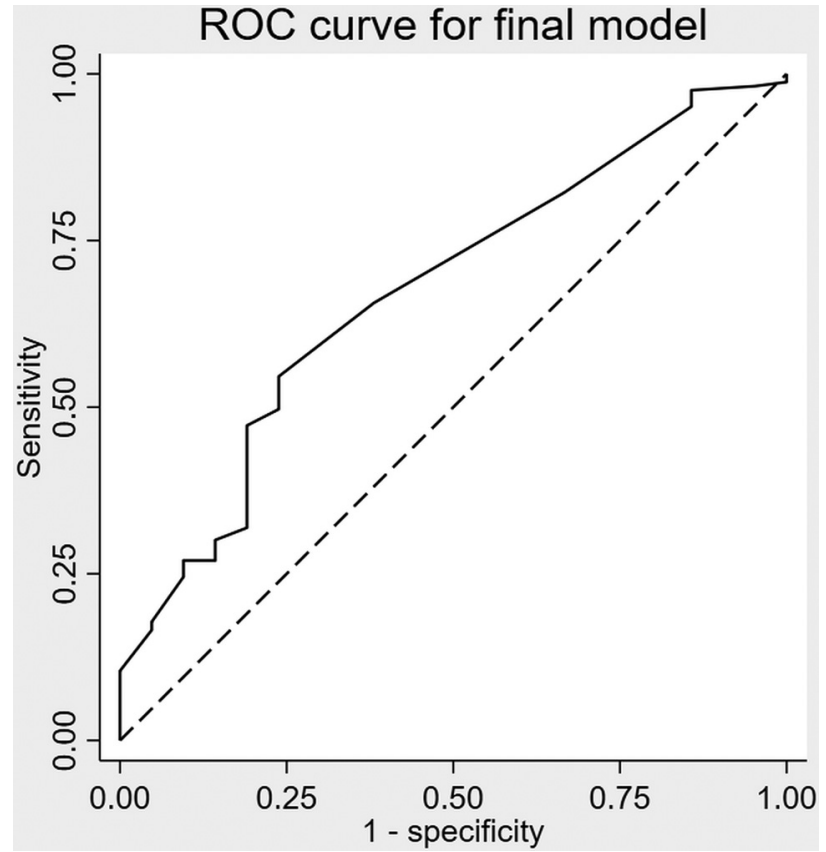

FIG 2. Receiver operating characteristic (ROC) curve of the final model of the forward selection stepwise regression.

Indicators will probably only be identified if all patients are randomized irrespective of their clinical presentation (airway, agitation, level of consciousness, and so forth), which is ethically challenging.

\section{CONCLUSIONS}

In this meta-analysis of randomized anesthesia trials, there are no clear factors that predict the risk of conversion to GA for a patient who seems amenable to EVT under CS. However, if a patient needs conversion to GA, the outcome is worse. These findings potentially support the use of primary GA in all patients before EVT, provided that it can be rapidly administered.

Disclosures: Claus Z. Simonsen—RELATED: Grant: Novo Nordisk Foundation.* Pia Löwhagen Hendén-UNRELATED: Employment: Sahlgrenska University Hospital, clinical work as an MD. Albert J. Yoo-UNRELATED: Consultancy: Cerenovus, Penumbra, Genentech; Grants/Grants Pending: Medtronic, Cerenovus, Penumbra, Stryker, Genentech*; Other: Insera Therapeutics, Comments: equity interest. Julian BöselUNRELATED: Grants/Grants Pending: Patient-Centered Outcomes Research Institute, Comments: participation in Patient-Centered Outcomes Research Institute, Award for SETPOINT2; Payment for Lectures Including Service on Speakers Bureaus: Medtronic, Boehringer Ingelheim, ZOLL Medical Corporation, Comments: speakers honoraria and travel support; Royalties: Thieme, Comments: book on neurocritical care, several book chapters; Travel/Accommodations/Meeting Expenses Unrelated to Activities Listed: Medtronic, Boehringer Ingelheim, ZOLL Medical Corporation. Mads Rasmussen — RELATED: Grant: Health Research Foundation of Central Denmark Region. *Money paid to the institution.

\section{REFERENCES}

1. Goyal M, Menon BK, van Zwam WH, et al; HERMES collaborators. Endovascular thrombectomy after large-vessel ischaemic stroke: a meta-analysis of individual patient data from five randomised trials. Lancet 2016;387:1723-31 CrossRef Medline

2. Lapergue B, Blanc R, Gory B, et al; ASTER Trial Investigators. Effect of endovascular contact aspiration vs stent retriever on revascularization in patients with acute ischemic stroke and large vessel occlusion: the ASTER randomized clinical trial. JAMA 2017;318:443-52 CrossRef Medline

3. Carrera D, Gorchs M, Querol M, et al; Catalan Stroke Code and Reperfusion Consortium (Cat-SCR). Revalidation of the race scale after its regional implementation in Catalonia: a triage tool for large vessel occlusion. J Neuroiinterv Surg 2019;11:751-56 CrossRef Medline

4. Campbell BC, van Zwam WH, Goyal M, et al; HERMES collaborators. Effect of general anaesthesia on functional outcome in patients with anterior circulation ischaemic stroke having endovascular thrombectomy versus standard care: a meta-analysis of individual patient data. Lancet. Neurol 2018;17:47-53 CrossRef Medline

5. Schonenberger S, Uhlmann L, Hacke W, et al. Effect of conscious sedation vs general anesthesia on early neurological improvement among patients with ischemic stroke undergoing endovascular thrombectomy: a randomized clinical trial. JAMA 2016;316:1986-96 CrossRef Medline

6. Lowhagen Henden P, Rentzos A, Karlsson JE, et al. General anesthesia versus conscious sedation for endovascular treatment of acute ischemic stroke: the ANSTROKE trial (anesthesia during stroke). Stroke 2017;48:1601-07 CrossRef Medline

7. Simonsen CZ, Yoo AJ, Sorensen LH, et al. Effect of general anesthesia and conscious sedation during endovascular therapy on infarct growth and clinical outcomes in acute ischemic stroke: a randomized clinical trial. JAMA Neurol 2018;75:470-77 CrossRef Medline

8. Brinjikji W, Murad MH, Rabinstein AA, et al. Conscious sedation versus general anesthesia during endovascular acute ischemic stroke treatment: a systematic review and meta-analysis. AJNR Am J Neuroradiol 2015;36:525-29 CrossRef Medline

9. Steinberg JA, Somal J, Brandel MG, et al. Site of occlusion may influence decision to perform thrombectomy under general anesthesia or conscious sedation. Neurosurg Anesthesiol 2019 Sept 17. [Epub ahead of print] CrossRef Medline

10. John S, Thebo U, Gomes J, et al. Intra-arterial therapy for acute ischemic stroke under general anesthesia versus monitored anesthesia care. Cerebrovasc Dis 2014;38:262-67 CrossRef Medline

11. van den Berg LA, Koelman DL, Berkhemer OA, et al; Participating centers. Type of anesthesia and differences in clinical outcome after intra-arterial treatment for ischemic stroke. Stroke 2015;46:1257-62 CrossRef Medline

12. Bekelis K, Missios S, MacKenzie TA, et al. Anesthesia technique and outcomes of mechanical thrombectomy in patients with acute ischemic stroke. Stroke 2017;48:361-66 CrossRef Medline

13. Davis MJ, Menon BK, Baghirzada LB, et al; Calgary Stroke Program. Anesthetic management and outcome in patients during endovascular therapy for acute stroke. Anesthesiology 2012;116:396-405 CrossRef Medline

14. Abou-Chebl A, Zaidat OO, Castonguay AC, et al. North American Solitaire Stent-Retriever Acute Stroke Registry: choice of anesthesia and outcomes. Stroke 2014;45:1396-1401 CrossRef Medline

15. Abou-Chebl A, Yeatts SD, Yan B, et al. Impact of general anesthesia on safety and outcomes in the endovascular arm of Interventional Management of Stroke (IMS) III trial. Stroke 2015;46:2142-48 CrossRef Medline

16. Schonenberger S, Henden PL, Simonsen CZ, et al. Association of general anesthesia vs procedural sedation with functional outcome among patients with acute ischemic stroke undergoing thrombectomy: a systematic review and meta-analysis. JAMA 2019;322:1283-93 CrossRef Medline

17. Flottmann F, Leischner H, Broocks G, et al. Emergency conversion to general anesthesia is a tolerable risk in patients undergoing mechanical thrombectomy. AJNR Am J Neuroradiol 2020;41:122-27 CrossRef Medline 